\title{
Femtosecond laser fabrication for the integration of optical sensors in microfluidic lab-on-chip devices
}

\author{
R. Osellame ${ }^{1}$, R. Martinez Vazquez ${ }^{1}$, C. Dongre ${ }^{2}$, R. Dekker ${ }^{2}$, H.J.W.M. Hoekstra ${ }^{2}$, \\ M. Pollnau ${ }^{2}$, R. Ramponi ${ }^{1}$, and G. Cerullo ${ }^{1}$ \\ ${ }^{1}$ Istituto di Fotonica e Nanotecnologie del CNR - Dipartimento di Fisica del Politecnico di \\ Milano, P.zza L. da Vinci 32, 20133 Milano, Italy \\ E-mail: Roberto.osellame@polimi.it \\ ${ }^{2}$ Integrated Optical MicroSystems, MESA+ Institute for Nanotechnology, University of \\ Twente, PO Box 217, 7500 AE Enschede, The Netherlands \\ E-mail: M.Pollnau@ewi.utwente.nl
}

\begin{abstract}
Femtosecond lasers enable the fabrication of both optical waveguides and buried microfluidic channels on a glass substrate. The waveguides are used to integrate optical detection in a commercial microfluidic lab-on-chip for capillary electrophoresis.
\end{abstract}

A lab-on-chip (LOC) is a device that squeezes onto a single glass substrate the functionalities of a biological laboratory, by incorporating a network of microfluidic channels, reservoirs, valves, pumps and micro-sensors [1]. It offers the capabilities of preparation, transport, reaction and analysis of very small volumes (nano- to picoliters) of biological samples. Its main advantages are high sensitivity, speed of analysis, low sample and reagent consumption and the possibility of measurement automation and standardization. The LOC concept has a huge application potential in many fields, ranging from basic science (genomics and proteomics), to chemical synthesis and drug development, point-of-care medical analysis and environmental monitoring. The next technological challenge of LOCs is direct on-chip integration of photonic functionalities, by manufacturing optical waveguides for sensing of biomolecules flowing in the microchannels. Such integrated approach has many advantages over traditional free space optical sensing, including compactness, sensitivity, enhanced device portability and the possibility of multipoint excitation. However, standard waveguide fabrication methods are planar multistep processes, which considerably complicate the LOC device production.

Femtosecond-laser induced refractive index modification is a powerful technique enabling direct, maskless three-dimensional fabrication of optical waveguides in glass [2], and appears to be particularly suited for their integration into LOCs. It allows to position optical waveguides (or more complex photonic devices such as splitters and interferometers) inside a pre-existing LOC without affecting the manufacturing procedure of the microfluidic part of the device, thus greatly simplifying the production process. In addition, femtosecond-laser irradiation of fused silica followed by chemical etching in HF solution allows the manufacturing of directly buried microfluidic channels [3], due to the enhanced (by up to two orders of magnitude) etching rate of the irradiated material with respect to the pristine one. This opens the possibility of using a single femtosecond laser system for the production and the integration of microfluidic channels and optical waveguides [see Fig. 1(a)].

In this work we demonstrate the fabrication, by femtosecond laser irradiation, of both high-quality optical waveguides and microfluidic channels on the same fused silica substrate. We also use the femtosecond laser to inscribe optical waveguides on a 
commercial LOC for capillary electrophoresis (CE), for the realization of compact, highly sensitive integrated optical sensors.
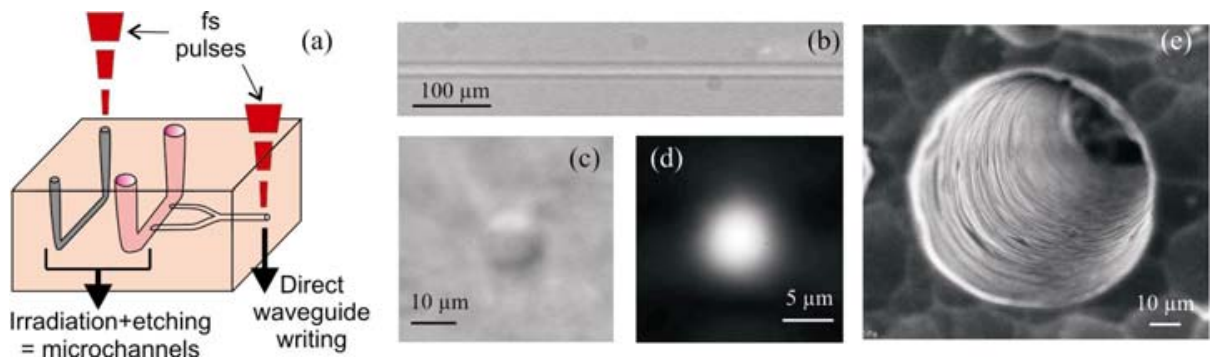

Fig. 1. (a) Conceptual scheme of the use of the femtosecond laser for the fabrication of optical waveguides and microfluidic channels; (b,c) top and end view of a femtosecond written waveguide;(d) waveguide mode at $532 \mathrm{~nm}$;(e) SEM image of a microfluidic channel obtained by femtosecond laser irradiation followed by chemical etching.

The femtosecond laser fabrication process employs a regeneratively amplified Ti:sapphire laser generating $150-\mathrm{fs}, 500-\mu \mathrm{J}, 800-\mathrm{nm}$ pulses at $1 \mathrm{kHz}$. A fraction of the beam is focused, by either a $20 \times(\mathrm{NA}=0.3)$ or a $50 \times(\mathrm{NA}=0.6)$ objective, at a depth ranging from $100 \mu \mathrm{m}$ to $500 \mu \mathrm{m}$ below the surface of the fused silica sample, which is moved perpendicularly to the beam propagation direction by a precision translation stage at a speed of $\approx 20 \mu \mathrm{m} / \mathrm{s}$. In order to produce modifications with a circular crosssection, the beam is astigmatically shaped by passing it through a cylindrical telescope [4]. The optical waveguides, fabricated using the $20 \times$ objective and $4 \mu \mathrm{J}$ pulse energy, are very uniform, have a nearly circular cross section with $10 \mu \mathrm{m}$ diameter and are single mode in the visible [see Figs. 1(b)-(d)]. The peak refractive index change is $1 \times 10^{-3}$. Propagation losses in the visible are $0.5 \div 1 \mathrm{~dB} / \mathrm{cm}$, which is generally a lower value than those obtained for waveguides integrated on LOCs by photolithographic techniques. The microfluidic channels are manufactured by higher intensity laser irradiation (pulse energy of $4 \mu \mathrm{J}$ through a $50 \times$ objective) and subsequent etching for 3 hours in an ultrasonic bath with a 20\% HF solution in water. With double side etching, channel lengths up to $3 \mathrm{~mm}$ with $100 \mu \mathrm{m}$ cross section are obtained [5]. The channels are directly buried and can be positioned at any depth with respect to the sample surface, in a three-dimensional fashion. Increased channel lengths, required for the LOC applications, can be obtained by a combination of iterative etching, concentration gradient and non-uniform sample irradiation. The quality of the microchannels is high, as shown in the SEM image of Fig.1e.

To test the applicability of our fabrication technique to real-world devices, we inscribed optical waveguides in a commercial LOC for CE (LioniX bv, the Netherlands), a schematic layout of which is shown in Fig.2(a). This chip has two crossing microchannels, that are responsible for the sample injection (channel going from reservoir 1 to 3 ) and for the electrophoretic separation (channel going from reservoir 2 to 4). Several optical waveguides have been inscribed perpendicular to the separation channel towards its end, to provide highly localized excitation for laserinduced fluorescence (LIF). A precise alignment procedure has been developed in order to have the $10 \mu \mathrm{m}$-diameter waveguides exactly crossing the 12 - $\mu \mathrm{m}$-high 
microfluidic channel. A low power green laser was coupled to a waveguide by a single mode optical fiber and the separation channel was filled with a solution of Rhodamine 6G. Figure 2(b) shows the yellow fluorescence excited by the green light coupled into the waveguide. The excitation is selective in space $(10 \mu \mathrm{m}$, as the waveguide diameter), indicating low light leakage out of the waveguide. In addition, the fluorescence covers the whole width $(50 \mu \mathrm{m})$ of the channel due to the low divergence of light coming from a waveguide with a numerical aperture below 0.1

As shown in Fig.2(a), the LIF signal is collected by an ultrahigh numerical aperture optical fiber $(\mathrm{NA}=0.5$ ) glued to the chip in correspondence to the excited portion of the microchannel in a $90^{\circ}$ geometry with respect to the exciting waveguide, thus strongly suppressing the excitation light background. The signal is detected by a photon counting photomultiplier after notch and interference filters, used to further reject the excitation light and chip autofluorescence. Measurements indicate a limit of detection of $40 \mathrm{pM}$, comparable to the best results obtained with standard free-space optics, with the clear advance of enhanced compactness and portability.

In conclusion, the femtosecond laser is a powerful tool for the fabrication of optical waveguides and microfluidic channels and their combination in optofluidic devices. It can be used to integrate optical excitation/detection in commercial LOCs without affecting their manufacturing process, strongly increasing device compactness and portability.

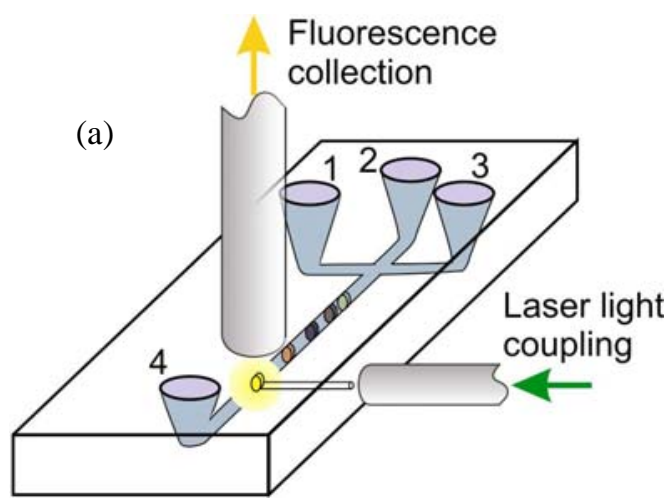

(b)

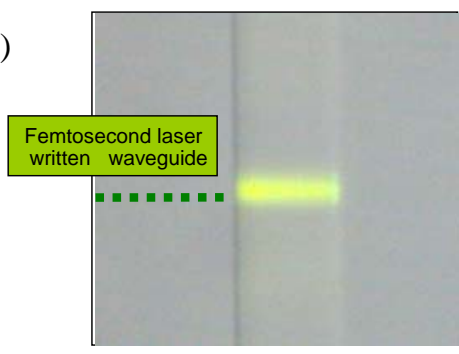

Fig. 2. (a) Schematic diagram of the commercial microfluidic chip with femtosecond laser inscribed optical waveguides; (b) fluorescence induced in the microchannel by the optical waveguide.

Acknowledgements. This work was funded by the European Commission, 6th FP STREP Project Contract No. IST-2005-034562 [Hybrid Integrated Biophotonic Sensors Created by Ultrafast laser Systems (HIBISCUS)].

1 G.M.Whitesides, Nature 442, 368-373 (2006).

2 K. M. Davis, K. Miura, N. Sugimoto and K. Hirao, Opt. Lett. 21, 1729-1731 (1996).

3 A. Marcinkevicius, S. Juodkazis, M. Watanabe, M. Miwa, S. Matsuo, and H. Misawa, Opt. Lett. 26, 277-279 (2001).

4 R. Osellame, S. Taccheo, M. Marangoni, R. Ramponi, P. Laporta, D. Polli, S. De Silvestri, and G. Cerullo, J. Opt. Soc. Am. B 20, 1559-1567 (2003).

5 R. Osellame, V. Maselli, R. Martinez Vazquez, R. Ramponi, and G. Cerullo, Appl. Phys. Lett. 90, 231118, 2007. 